\title{
Correlative Imaging to Probe Heterogeneity of Cross-Linked Gelatin
}

\author{
J.-B.D. Green ${ }^{1}$, R. Lin ${ }^{1}$, M.E. Francis-Sedlak ${ }^{2}$, J.P. DiOrio ${ }^{1}$, M. A. Murphy ${ }^{1}$, S.C. Strathmann ${ }^{1}$, \\ L.L. Stojanovic ${ }^{1}$ and J.R. Mantei ${ }^{1}$
}

1. Baxter Technology Resources, Round Lake, Illinois USA

2. Baxter Cellular Therapies, Round Lake, Illinois USA

Wound management products used to control bleeding play an essential role in critical care treatments especially in trauma and surgical settings. Gelatin based products are available as a topical hemostatic agent and are used to control bleeding when conventional surgical procedures are ineffective or impractical [1]. Their hemostatic properties result from combining a cross-linked gelatin matrix with thrombin to produce a highly viscous gel that effectively controls bleeding In the work presented here, differences in the cross-linked gelatin matrix induced by unique cross-linkers were characterized, and the mechanical properties were studied with correlative microscopic techniques.

These model gelatin matrices were formed with "in vitro" systems in conjunction with anti-coagulated whole blood (Figure A), labeled fibrinogen solution (Figure B) or blood plasma (Figures C, D) to form mechanically robust clots which emulate the clots formed during hemostasis. The three dimensional structure of these clots was examined with both confocal fluorescent microscopy (Nikon A1-R) and micro computerized tomography (Micro-CT: SkyScan 1172). The whole blood and plasma clots were also sectioned with vibratome, and these sections were further examined with confocal fluorescence microscopy, scanning electron microscopy (SEM: JEOL 7600), and atomic force microscopy (AFM: Veeco NanoScope V). The SEM clots were fixed in glutaraldehyde, dehydrated in a graded ethanol series and dried from hexamethyldisilazane (HMDS). The plasma clots were not fixed and the fibrinogen clots were fixed in paraformaldehyde.

The cross-linker does not seem to have made a difference in the three dimensional packing structure of the gelatin granules. The confocal data revealed that in the case of one cross-linking agent, the cross-linking density was uniformly distributed throughout the gelatin (Figure D), while in the case of the other cross-linker (Figure C), the cross-linking was predominantly localized at the edge of the gelatin granules. This heterogeneity was further probed with the AFM by performing elastic modulus measurements at different locations within the gelatin granules. Figures $\mathrm{E}$ and $\mathrm{F}$ show some representative force curves and corresponding histograms of elasticity measurements taken from the centers of two granules. The results indicate that the cross-linker type can have a significant impact on the viscoelastic properties of individual gelatin granules including intra-granule homogeneity.

References:

[1] C. Mehmet, et al., J. Card. Surg. 18 (2003) 486 and Ann. Thorac.Surg. 69 (2000) 1376 
Fig. A. SEM image of a vibratome section of a fixed gelatin clot formed with whole blood.

Fig. B. Confocal image of a fixed clot formed from gelatin cross-linked with cross-linker A and with Alexa Fluor 647 fluorescently labeled fibrinogen.

Fig. C. Confocal image of an un-fixed clot formed from plasma and gelatin cross-linked with crosslinker A, with inset line scan.

Fig. D. Confocal image of an un-fixed clot formed from plasma and gelatin cross-linked with crosslinker B, with inset line scan.

Fig. E. Representative AFM force curves for gelatin cross-linked with the cross-linkers A and B, along with Hertzian fits to the data.

Fig. F. Histogram of elastic moduli corresponding to the center of the gelatin granules.

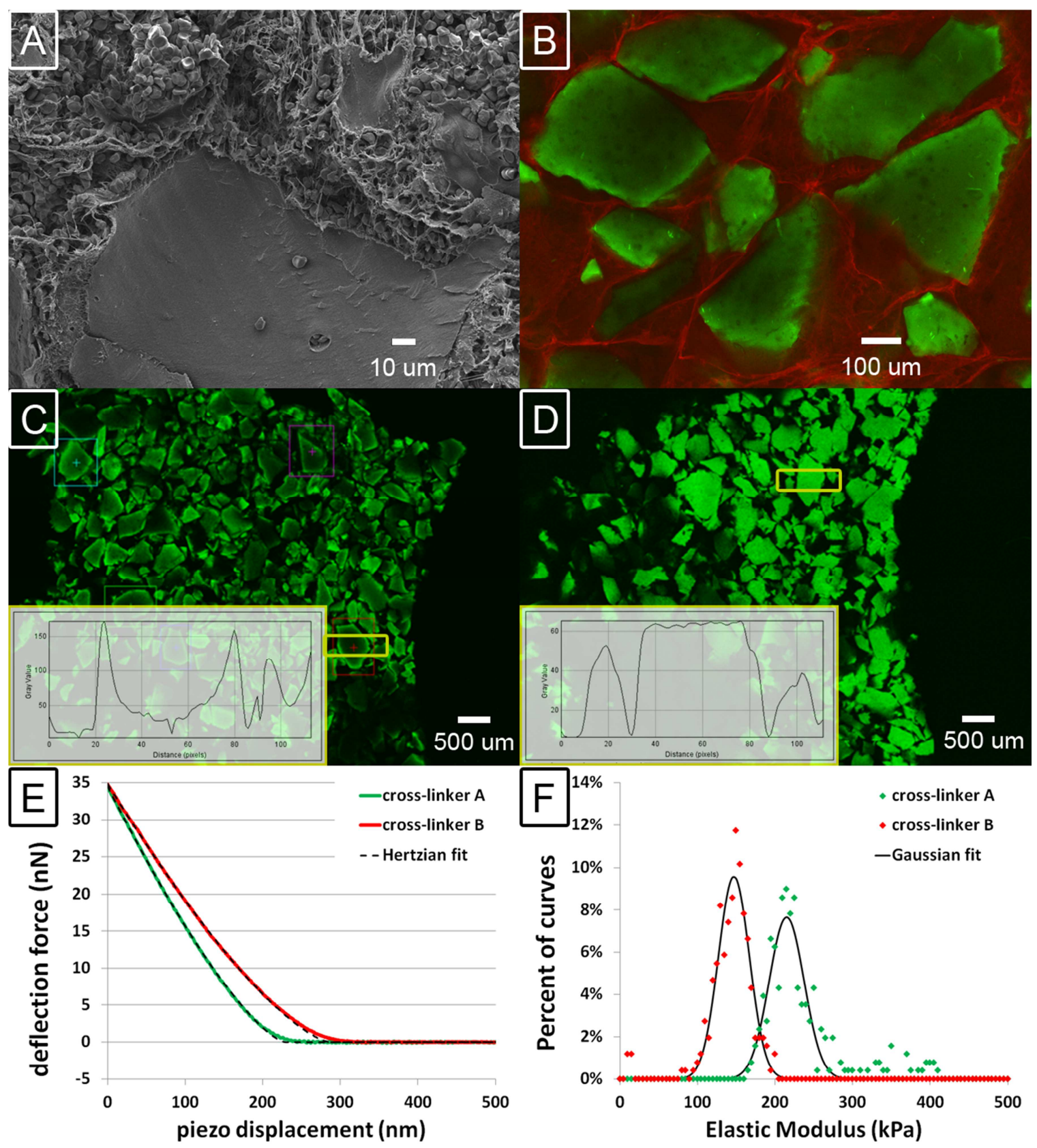

